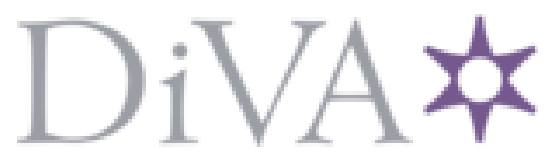

http://www.diva-portal.org

\title{
Preprint
}

This is the submitted version of a paper published in British Journal of Dermatology.

Citation for the original published paper (version of record):

Gaele, K., Henriksson, M., Schmitt-Egenolf, M. (2016)

Evaluating equality in psoriasis healthcare: a cohort study of the impact of age on prescription biologics.

British Journal of Dermatology, 174(3): 579-587

http://dx.doi.org/10.1111/bjd.14331

Access to the published version may require subscription.

N.B. When citing this work, cite the original published paper.

Permanent link to this version:

http://urn.kb.se/resolve?urn=urn:nbn:se:umu:diva-112179 


\section{Evaluating equality in psoriasis healthcare: a cohort study of the impact of age on prescription biologics}

Kirk Geale ${ }^{1,2}$, Martin Henriksson ${ }^{2,3}$, Marcus Schmitt-Egenolf ${ }^{1}$

${ }^{1}$ Department of Public Health and Clinical Medicine, Dermatology, Umeå University, Umeå, Sweden; ${ }^{2}$ PAREXEL International, Stockholm, Sweden; ${ }^{3}$ Department of Medical and Health Sciences, Linköping University, Linköping, Sweden

Corresponding author: Marcus Schmitt-Egenolf, Dep. of Public Health and Clinical Medicine, Dermatology, Umeå University, SE-901 87 Umeå, Sweden

+46 90785 2875, marcus.schmitt-egenolf@umu.se

www.derma.org

Word count: 3925, Table count: 5, Figure count: 3

Funding sources: PsoReg receives financial support from the Swedish Board of Health and Welfare, the Swedish Association of Local Authorities and Regions and the Västerbotten County Council. Sponsors had no access to the data. Data collection, study design, interpretation, and analyses have been carried out independently by the authors.

Conflict of interest disclosure: KG and MH disclose that they were employed by PAREXEL International during the time when the manuscript was written, and have no other disclosures. MSE has no conflicts of interest to disclose. 


\section{What is already known on this topic:}

- Inequalities in healthcare have been identified in many contexts, prompting political policy to focus on their reduction

- Older patients may not have the same opportunities to access healthcare as younger patients with similar clinical profiles

- This trend has been shown in the majority of research in rheumatoid arthritis, a field that uses many of the same biologic treatments as psoriasis

\section{What this study adds:}

- The first examination of the relationship between age and access to biologic medication in psoriasis

- Patients are less likely to receive prescriptions for biologic treatments as they age. An increase of 30 years in age leads to a $61.3-67.6 \%$ decrease in the likelihood of receiving access to biologic treatments after controlling for sex, comorbidities, education level, BMI, and disease severity

- Evidence of age inequality in access to biologic treatments for psoriasis patient 


\begin{abstract}
Background: Inequality in healthcare has been identified in many contexts. To the best of our knowledge, this is the first study investigating age inequality in the form of prescription patterns of biologics in psoriasis care.
\end{abstract}

Objective: To determine whether psoriasis patients have equitable opportunities to receive biologic medications as they age. If patients do not receive equitable treatment, a subsequent objective is to determine the magnitude of the disparity.

Methods: A cohort of biologic-naïve psoriasis patients were analysed using Cox proportional hazard models to measure the impact of each additional year of life on the likelihood of initiating biologic treatment, after controlling for sex, body mass index, comorbidities, disease activity, and education level. A supporting analysis used a non-parametric graphical method to study the proportion of patients initiating biologic treatment as age increases, after controlling for the same covariates.

Results: The Cox proportional hazards model results in a hazard ratio of a one year increase in age of 0.963 to 0.969 depending on calendar year stratification, which implies that an increase in age of 30 years corresponds to a reduced likelihood of initiating biologic treatment by 61.3$67.6 \%$. The estimated proportion of patients initiating biologic medication is always decreasing as age increases, at a statistically significant level.

Conclusions: Psoriasis patients have fewer opportunities to access biologic medications as they age. This result was shown to be applicable at all stages in a patient's life course and was not only restricted to the elderly, although it implies greater disparities as the age difference between patients increases. These results show that inequality in access to biologic treatments due to age is prevalent in clinical practice today. Further research is needed to investigate the extent to which this result is influenced by patient preferences. 


\section{Introduction}

According to a recent systematic review of psoriasis epidemiology approximately $2-4 \%$ of the population in Western countries are affected by psoriasis, with general increases in prevalence as the distance from the equator increases ${ }^{1}$. This systematic review shows that Scandinavia in particular has a relatively high prevalence of psoriasis. Moderate to severe psoriasis requires regular systemic treatments, compared to mild psoriasis which can be sufficiently treated with topical applications only. Many patients with moderate to severe psoriasis require treatment with biologics when other methods do not provide sufficient response. Biologics are medications that either target T-cells or block immune system proteins, the first of which was approved for the treatment of psoriasis in Sweden in 2004. Biologic treatments currently available in the Swedish formulary include adalimumab, etanercept, infliximab, secukinumab, and ustekinumab; all of which have high efficacy and high acquisition costs.

High quality, representative data is necessary to draw reliable conclusions from which practitioners can practice evidence based medicine. Population-based registries provide favourable data conditions for studying questions of inequality, as they can evaluate clinical practice for a given population in a real-world environment. The advantages of registries have been exploited in previous research focusing on health inequalities ${ }^{2-4}$. The Swedish psoriasis population-based quality registry, PsoReg, contains prescription records and sociodemographic data for patients with moderate to severe psoriasis.

The PsoReg data enables us to study the effects of age equality in healthcare access in clinical practice, complimented by the fact that biologics are effective treatments with clear prescription guidelines. The present study examines the age gradient in prescription rates of biologics for psoriasis patients after accounting for disease severity.

There has been little improvement in recent history in levels of inequality: During the 1980s and 1990s, socioeconomic inequalities in self-reported health did not improve over 10 European countries $^{5}$. In response to these and other similar findings, the WHO initiated a large research project in 2005 to identify and determine causes of social-based health inequalities with the purpose of promoting political action based on the results ${ }^{6}$. It was concluded that reducing health inequalities is a matter of social justice. One area of inequality research has focused on access to healthcare, where age is a determinant of interest ${ }^{7-9}$.

The proportion of elderly persons will grow rapidly in the near future. For example, it is estimated that the percentage of the population over age 65 in the United States will grow from $13.7 \%$ in 2012 to $20.3 \%$ in 2030 , equivalent to a size of approximately 73 million people ${ }^{10}$. As the size of this age group grows, the question of how the health care system treats patients as they age becomes increasingly relevant. However, studies investigating age and access to healthcare are uncommon. In light of the relevant research that has been published, the results have not necessarily lead to changes in clinical practice - this was exemplified by the recent report describing a lack of action following the identification of under-treated elderly cancer patients ${ }^{11}$. It is also well known that older patients are regularly excluded from clinical trials; an important source of information from which physicians make treatment decisions. A systematic 
review found that one in five medical prescriptions to patients over age 65 should be deemed inappropriate according to the Beers criteria in primary care ${ }^{12}$.

\section{Methods}

\section{Data}

Data used in the present study were extracted from PsoReg ${ }^{13}$, a national quality registry consisting of patients with moderate to severe psoriasis receiving systemic treatment from a specialist in Sweden, combined with ICD-10 diagnosis codes from the national patient registry ${ }^{14}$, and annual education data from the Longitudinal Integration Database for Health Insurance and Labour Market Studies ${ }^{15}$. Education data is available for a shorter timespan than the other two aforementioned registries (until 2009) and therefore defines the end of the time horizon used in this study. The psoriasis registry's coverage of patients receiving biologic medication prescriptions is approximately $65 \%$, where coverage is calculated as the number of patients enrolled in the psoriasis registry divided by the total number of eligible patients. The statistical analyses included a total of 1465 biologic naïve patients (Figure 1) in PsoReg registered between 2007 and 2009. One patient was removed due to data entry error. The dataset contained a total of 259 events where biologic medication was initiated. Biologic initiation is defined as the first time a patient receives a prescription for any biologic medication, and can therefore only occur once per patient. The average time at risk of initiating a biologic medication was 0.90 years for patients receiving biologic prescriptions during the study period and 5.18 years for patients with no biologic prescription by the end of the study period.

The occurrence of a biologic initiation, and corresponding time to initiation, were principal aspects of the analyses conducted. Time at risk of a biologic prescription began at the date of inclusion in the psoriasis registry. If a patient initiated treatment at the first registered contact date (implying 0 days at risk), the patient was assumed to have 1 day at risk to allow these patients to be included in the analysis. In the absence of information about a biologic prescription, it was assumed that no biologic prescription was given. This is reasonable since the registry's purpose is to evaluate biologic treatments, and participating clinics are aware that biologic medications must be registered.

The covariates used in the analyses were age, sex, education level, Charlson comorbidity index $(\mathrm{CCl})$, body mass index (BMI), presence of psoriatic arthritis, and the psoriasis area, and severity index (PASI). Differences in variable means or proportions can be observed between patients who initiated biologic treatment and those that did not (Error! Reference source not found.). In contrast, covariates appear to be fairly well balanced between the years of entry into the psoriasis registry (Table 2), with the exceptions of the proportion treated - this can be explained by the fact that those enrolled in earlier years have had more time at risk of receiving a biologic prescription, since the end of study date is the same for all patients, regardless of enrolment date.

In the same fashion as the presence of a biologic prescription, psoriatic arthritis was considered to be absent if its presence was not explicitly recorded at baseline. In the dataset, if BMI or PASI was missing for any observation, it was missing for all observations for that patient. As such, a 
missing value for BMI or PASI resulted in the removal of the patient. No imputation was conducted.

Some variables used in the statistical analyses were selected based on treatment criteria. In order to be eligible for treatment with biologic interventions according to British guidelines ${ }^{16}$, patients should have severe disease as defined by a PASI score and a Dermatology Life Quality Index (DLQI) score of 10 or more, with severe psoriasis for six months or more. The guidelines also specify that patients should fulfil at least one of seven additional criteria relating to toxicity or unresponsiveness to standard therapies, persistent inpatient care, comorbidities, lifethreatening psoriasis, or presence of psoriatic arthritis. Of these treatment criteria, PsoReg includes both disease severity measures (PASI and DLQI scores) as well as one of the secondary eligibility criteria (presence of psoriatic arthritis). Because PASI and DLQI both measure disease severity, and PASI is more strongly associated with the decision to prescribe biologic treatment than DLQI ${ }^{17}$, only PASI was included in the statistical analyses to avoid multicollinearity.

Patient's treatment preference is not recorded in PsoReg. In an attempt to control for patients' treatment preference, patients' level of education was included as a covariate. It has been well documented that education level is associated with increased participation level in treatment decisions, together with sex and age ${ }^{18}$. Further, higher levels of education are theoretically and empirically linked with improved health outcomes ${ }^{19 ; 20}$. As biologic medications have been shown to be safe and effective in clinical trials, and are viewed as such by the scientific community ${ }^{21}$, it could be argued that patients with relatively higher levels of education may have more influence on their prescribed treatments (increased participation) and are more aware of biologics as novel and effective treatments (increased health literacy). Based on this reasoning, education level may be a reasonable proxy measure for treatment preference, and it is hypothesised that education level is associated with an increased rate of biologic initiation. Education level was included by modelling three categorical variables for those achieving less than, up to, or beyond high school ${ }^{\mathrm{a}}$ level qualifications.

Physicians may be less willing to prescribe treatments targeting the immune system, such as biologics, for patients with additional comorbidities. Depending on the comorbidity, patients may be contraindicated for biologic treatment. Therefore, ICD-10 diagnosis codes for patients in PsoReg were collected from the Swedish national patient registry from 2007 to the latest registered date. A longitudinal comorbidity variable (summed over all years before baseline) was created based on the $\mathrm{CCl}^{22}$ using the ICD-10 groupings described by Quan and colleagues $^{23}$, and coded using the algorithm described by Turner and Burchill ${ }^{24}$. Psoriatic arthritis as a comorbid condition is included separately because it is not included in the $\mathrm{CCl}$, but is particularly relevant for the patient population under investigation.

\section{Statistical analyses}

The control variables included in the analyses were selected to reflect the factors that impact physicians' prescription decisions. These variables aim to capture the effects of disease

\footnotetext{
a We refer to high school as the Swedish education level of "gymnasial utbildning," which translates to secondary education. This level of education typically lasts for three years.
} 
severity, comorbidities, observed treatment disparities between males and females, and patient preference on physicians' treatment decisions. The remaining factor included in the models is age, which we aim to examine for the presence of inequality. By including other variables that are related to prescription decisions, our aim is to remove the effects of the variables correlated with age in order to estimate the unbiased effect of age only. We define a result showing a statistically significant effect of age on the decision to initiate biologic treatment, after controlling for these covariates, as evidence of age inequality. As described above, age alone should not impact treatment decisions, other than through correlated variables such as comorbidities.

A survival analysis was conducted using a Cox proportional hazards model where exposure time, censoring, and covariates were prepared and interpreted according to the description of the data provided above. To account for increases in medical knowledge of biologics as calendar time $^{b}$ passes, three separate survival models were estimated, stratified by year. This accounts for the fact that patients entering the registry in 2007 are less likely to initiate a biologic treatment after a given amount of time passes than a patient entering the registry in 2009 , since both patients and physicians are more likely to be familiar with biologics as calendar time passes. The assumption of proportional hazards was evaluated for appropriateness after the estimation of each model. The survival analysis was conducted in Stata version 11.2.

A second analysis was conducted using a non-parametric kernel regression. The dependent variable was the initiation of a biologic medication, and one observation was included per patient. Patients with no biologic medication were assigned the last, and therefore oldest, age observed in the registry. For patients receiving biologic treatment, age was measured at the date the biologic prescription occurred. Two regressions were conducted: the first regressed biologic initiation on age only, and the second used all covariates described previously, corresponding to those in the survival analysis. The regressions were not stratified by calendar year since the estimates were almost indistinguishable between years. In both regressions, the non-parametric analyses show the estimated proportion of patients initiating biologic treatment decreases as age increases. Intrinsic to the nature of nonparametric kernel regressions, the estimates generated must be presented graphically instead of through a single parameter value as in survival analysis or parametric regression. These non-parametric estimation was implemented in $\mathrm{R}$ version 3.1.1 using the $\mathrm{np}$ package ${ }^{25}$ using local linear regression for mixed data types.

The survival analysis and the non-parametric analyses are complimentary, but measure different things. The classic survival analysis gives the difference in risk of receiving a biologic prescription for patients one year apart in age. The non-parametric kernel regressions estimate the effect of age on the proportion of patients receiving a biologic treatment.

\footnotetext{
${ }^{\mathrm{b}}$ We distinguish calendar time, i.e. January 1 , with survival time (where patients begin at time 0 on the day they are registered, regardless of calendar time).
} 


\section{Results}

\section{Proportion of patients initiating biologic treatment}

The non-parametric regression depicts a decreasing proportion of patients initiating biologic treatment as age increases in the simple regression on age only (Error! Reference source not found.), as well as in the regression where all covariates are included (Figure 3). For the fully specified regression, the estimation presented is the partial regression plot for proportion of patients initiating biologic treatment, on age. The solid centre line represents the estimate and the dotted lines give the corresponding 95\% confidence interval bounds.

The results of both regressions show an inverse relationship between biologic initiation and age. After age 40, the age gradient in the simple regression is noticeably steeper than in the fully specified regression. The interpretation of the age gradient in the fully specified regression is that the proportion of patients initiating biologic treatment decreases for each year gained in age, after controlling for the effects of covariates. The proportion of patients initiating biologic treatment is strictly decreasing across the total age range. Age is statistically significant in both regressions in a non-parametric context ${ }^{26 ; 27}$, where $p<0.001$.

\section{Time to biologic treatment initiation}

The Cox proportional hazards models, controlling for covariates (Table 3-Table 5), result in highly significant hazard ratios for age of $0.963,0.968$, and 0.969 in the 2007, 2008, and 2009 stratifications, respectively. In comparison, the hazard ratio of age unadjusted for covariates is $0.961,0.965$, and 0.967 , indicating a robust result even when covariates are not included. Since age is measured in years, the result implies that the chance of initiating a biologic treatment for a given patient is between 3.1-3.9\% lower, depending on stratification year, compared to a patient one year younger. When evaluating a one unit change of 30 years instead of one year in the proportional hazards model, we find a hazard ratio of $0.324,0.379$, and 0.387 over the same stratifications; indicating substantially lower chances of initiating a biologic prescriptions by 61.3$67.6 \%$ compared to a patient 30 year younger.

The assumption of proportional hazards was tested using Schoenfeld residuals and through visual inspection, where the hypothesis of proportional hazards could not be rejected at an alpha level of $5 \%$ over all parameters and for each individual parameter for stratification years 2007 and 2009. In 2008, the indicator variable for education completed up to high school failed both the statistical test of proportional hazards, as well as a visual inspection of the log-log survival plot. Therefore, this variable was included as a time-varying coefficient in the 2008 stratification by interacting it with the natural logarithm of time.

Similar to age, PASI is highly significant in all stratifications, where the hazard ratio is greater than one as hypothesized. In the 2008 stratification, education level is significant for the group completing high school at an alpha level of $10 \%$ and education level greater than high school is significant at a $5 \%$ level (less than high school is the reference group). As expected, both hazard ratios are greater than one, and the hazard of education greater than high school is larger than the hazard of completion of high school. This implies a stronger effect of higher levels of education on the initiation of a biologic treatment. Psoriatic arthritis patients are more 
likely to initiate biologic treatment, a statistically significant result in the 2008 and 2009 stratifications. The increased hazard for males in the 2009 stratification, significant at the $10 \%$ level, is consistent with previous research ${ }^{28}$ regarding the initiation of biologics. BMI is significant in the 2008 stratification at the $10 \%$ level. While BMI may not have a direct relationship to the initiation of biologic treatment, it is positively correlated with obesity and comorbidities, so a hazard ratio greater than one was expected for BMI. Increased levels of comorbidities also reflect a higher chance of a biologic prescription.

\section{Discussion}

The proportion of patients initiating biologic treatment is decreasing with age, as is the hazard rate of initiating a biologic treatment, after controlling for the effects of sex, education, comorbidities, BMI, and disease severity. The result is also clinically significant: the survival analyses shows that for every thirty years gained in age, a patient is between $61.3 \%$ and $67.6 \%$ less likely to initiate biologic treatment. If we assume that one generation spans 30 years, this implies that a mother has less than half the chance of initiating a biologic treatment than her daughter due to her age. Aside from age, PASI, and psoriatic arthritis are the most consistently statistically significant variables associated with the initiation of biologic treatment. PASI and psoriatic arthritis are clear disease activity markers that can be expected to have a strong influence on the initiation of effective medications such as biologics.

Despite similar disease profiles between younger and older patients after controlling for covariates, patients may receive inferior access to healthcare as they age. The conclusions drawn from the survival analyses are consistent with the majority of research in the area of rheumatoid arthritis $(\mathrm{RA})^{29-31}$, where a similar range of biologic treatments are available.

Physicians operate in challenging environments burdened by budget constraints and the high acquisition cost of biologic treatments. Biologics are effective treatments for patients with moderate to severe psoriasis, and should be available to all eligible patients. That patients receive significantly less opportunity to receive access to effective healthcare as they age is noteworthy. However, different phases of life may be intrinsically related to particular patient needs that cannot be disentangled from age given the data available. The results in this paper should be used to describe and discuss the treatment landscape but not to pass judgment on the behavior of prescribing physicians. Patients' preference need to be investigated as it is possible that many older patients, particularly with only moderate psoriasis, do not want to receive systemic treatments. Further, due to a lack of high quality trial-based safety data on biologics in older populations, physicians may be cautious in prescribing biologic medications to this group.

Although the age inequality is most evident when comparing young and old patients, the relationship between age and biologic initiation is also applicable to young patients - for every year increase in age, regardless of the reference age, patients are less likely to initiate a 
biologic treatment. Reduced access to healthcare resources has clear implications for patient outcomes. As such, prescribing physicians must be aware of, and act upon, health inequalities for vulnerable patient groups such as the elderly. This study illustrates the challenges patients, as well as clinicians, face in contemporary clinical practice. Both political and academic work has made it clear that reducing inequality in healthcare must be a goal of modern societies.

\section{Study strengths and limitations}

The present study did not address the possibility of selection bias for patients included in the registry. Increasingly robust conclusions will be possible as the registry sample size grows, and correspondingly the number of patients initiating biologics.

Missing or unknown values for biologic prescriptions and psoriatic arthritis respectively were assumed to take particular values based on logical reasoning and argumentation. Since the true values of these parameters are not known, the assumptions made may affect the results of the analysis. Removing the patients in question did not result in different conclusions. Patient preference is an important determinant of contemporary clinical decision making that we have aimed to capture in this study through the proxy measure of education. However, this is not a direct measure of patients' preference for, or knowledge of, biologic treatment. As discussed earlier, no direct measures of patient preference are captured in the registry data. Comorbidities are captured in this study through the psoriatic arthritis variable and the $\mathrm{CCl}$ variable. There may be other comorbidities not captured in either of these variables that play an important role in treatment decisions for psoriasis patients. As the number comorbidities is known to be increasing with patient age, this aspect of the analysis would ideally be more precise. Finally, not all secondary biologic prescription criteria are available in the registry and are not included in the regressions.

The current study analyses treatment decisions for psoriasis patients. While the registry data used is of high quality, it may be questioned whether this result is generalizable to other disease areas or dimensions of access to healthcare. Findings in the field of RA corroborate the results from this study, indicating consistency to a similar disease area. Further, we expect that the result of reduced access to healthcare for older patients, interpreted in other ways than prescription patterns, is likely to remain consistent with the current study since the behaviour of prescribing physicians is unlikely to change as all healthcare resources are a time and resource burden. The magnitude of the effect, however, may vary across different facets of access to healthcare.

The primary strength of this study is the ability to study prescription patterns for access to biologic medications in a population-based cohort using data that has been collected on various aspects of patients' lives that pertain to prescription patterns. The fact that biologic treatments have been available only in the past decade means that this case-study evaluates access to healthcare in contemporary clinical practice, while still providing a window of time that is long enough for the evaluation to include many patients that get the event of interest. This is, to the best of our knowledge, the first study of age inequality in psoriasis.

A second strength is that the registries used in this study contain variables that allow us to study not only the unadjusted relationship between prescriptions and age, but also to account for 
important factors that should determine biologic prescription initiation, such as demographics, disease severity, patient preference, and comorbidities. The psoriasis registry also provides information on previous biologic prescriptions, enabling us to examine only biologic-naïve patients.

\section{Future research}

This study has used population-based registries to present the first findings evaluating the presence of inequality in clinical practice in psoriasis. To account for patient preference, we have used education level as a proxy. However, experimental research is needed to directly address the question of both patient and clinician preference in the prescription of biologic medications relating to the age of the patient.

There is also ample opportunity to extend these results by quantifying the effects of healthcare access, measured by prescription patterns or otherwise, on patient outcomes. By linking established inequities to differences in patient outcomes, questions such as whether a particular inequality generates clinically meaningful reductions in health outcomes can be addressed. In psoriasis and similar diseases that do not affect mortality directly, outcomes measured by quality of life are particularly interesting.

\section{Conclusions}

The age gradient of initiating biologic treatments slopes downward and the chance of initiating biologic treatment decreases as patients age. As clinics face increasingly demanding budget environments, spending on therapies with high acquisition costs such as biologics can be limited. Therefore, it is necessary to investigate the equitable distribution of these scarce resources in clinical practice. This study provides the first analysis and results of age inequality in psoriasis healthcare, which indicate that patients who appear to be similar in many important ways except for age have different levels of access to biologic treatments. Given these results, improvements can be made to reduce inequities in contemporary clinical practice.

\section{Footnotes}

The authors would like to thank the patients and health care professionals contributing to the advancement of clinical practice through participation in quality registries such as PsoReg, as well as David Hägg for his support with queries regarding the PsoReg database.

Authorship: All authors contributed to the preparation and review of the manuscript, and to the interpretation of the data and results. KG drafted the manuscript and conducted the statistical analyses. All authors approved the final version.

Ethical approval: This research was done in adherence to the Declaration of Helsinki. Patients were recruited after that informed consent was obtained. Both data and consent was gathered electronically, to assure an effective logistic in this nationwide project. The Umeå Ethical Review Board, Sweden approved the project and the procedure for patient consent. 


\section{References}

1. Parisi R, Symmons DP, Griffiths CE et al. Global epidemiology of psoriasis: a systematic review of incidence and prevalence. Journal of Investigative Dermatology 2012; 133: 37785.

2. Addo J, Bhalla A, Crichton S et al. Provision of acute stroke care and associated factors in a multiethnic population: prospective study with the South London Stroke Register. Bmj $2011 ; 342$.

3. Coleman MP, Forman D, Bryant $\mathrm{H}$ et al. Cancer survival in Australia, Canada, Denmark, Norway, Sweden, and the UK, 1995-2007 (the International Cancer Benchmarking Partnership): an analysis of population-based cancer registry data. The Lancet 2011; 377 : 127-38.

4. Smith LK, Budd JL, Field DJ et al. Socioeconomic inequalities in outcome of pregnancy and neonatal mortality associated with congenital anomalies: population based study. Bmj $2011 ; 343$.

5. Kunst $A E$, Bos $V$, Lahelma $E$ et al. Trends in socioeconomic inequalities in self-assessed health in 10 European countries. International Journal of Epidemiology 2005; 34: 295-305.

6. Marmot M. Social determinants of health inequalities. The Lancet 2005; 365: 1099-104.

7. Fitzpatrick AL, Powe NR, Cooper LS et al. Barriers to health care access among the elderly and who perceives them. American Journal of Public Health 2004; 94: 1788.

8. Goins RT, Williams KA, Carter MW et al. Perceived barriers to health care access among rural older adults: a qualitative study. The Journal of Rural Health 2005; 21: 206-13.

9. Sudore RL, Mehta KM, Simonsick EM et al. Limited literacy in older people and disparities in health and healthcare access. Journal of the American Geriatrics Society 2006; 54: 7706.

10. Ortman JM, Velkoff VA, Hogan H. An aging nation: the older population in the United States.

11. Lawler M, Selby P, Aapro MS et al. Ageism in cancer care. BMJ: British Medical Journal $2014 ; 348$.

12. Opondo D, Eslami S, Visscher S et al. Inappropriateness of medication prescriptions to elderly patients in the primary care setting: a systematic review. PloS one 2012; 7 : e43617.

13. Schmitt-Egenolf M. PsoReg-The Swedish Registry for Systemic Psoriasis Treatment. Dermatology 2007; 214: 112-7.

14. Socialstyrelsen. Patientregistret database. 2010.

15. Statistics Sweden. Longitudinell integrationsdatabas för sjukförsäkrings- och arbetsmarknadsstudier. 2009. 
16. Smith $\mathrm{CH}$, Anstey AV, Barker JNWN et al. British Association of Dermatologists guidelines for use of biological interventions in psoriasis 2005. British Journal of Dermatology 2005; 153: $486-97$.

17. Hägg $D$, Sundström $A$, Eriksson $M$ et al. Decision for biological treatment in real life is more strongly associated with the Psoriasis Area and Severity Index (PASI) than with the Dermatology Life Quality Index (DLQI). Journal of the European Academy of Dermatology and Venereology 2014.

18. Say R, Murtagh M, Thomson R. Patients' preference for involvement in medical decision making: a narrative review. Patient education and counseling 2006; 60: 102-14.

19. Grossman M, Kaestner R. Elfects ol Education on Health. The social benefits of education 1997; 12: 69.

20. Paasche-Orlow MK, Wolf MS. The causal pathways linking health literacy to health outcomes. American journal of health behavior 2007; 31: S19-S26.

21. Sterry W, Barker J, Boehncke $\mathrm{W}$ et al. Biological therapies in the systemic management of psoriasis: International Consensus Conference. British Journal of Dermatology 2004; 151: 3-17.

22. Charlson ME, Pompei $\mathrm{P}$, Ales $\mathrm{KL}$ et al. A new method of classifying prognostic comorbidity in longitudinal studies: development and validation. Journal of chronic diseases 1987; 40: 373-83.

23. Quan $\mathrm{H}$, Sundararajan $\mathrm{V}$, Halfon $\mathrm{P}$ et al. Coding algorithms for defining comorbidities in ICD-9-CM and ICD-10 administrative data. Medical care 2005; 1130-9.

24. Turner, K. and Burchill, C. (5-11-2004) Charlson ICD-10 SAS Macro [WWW document]. URL http://mchp-appserv.cpe.umanitoba.ca/Upload/SAS/_CharlsonICD10.sas.txt

25. Hayfield T, Racine JS. Nonparametric econometrics: The np package. Journal of statistical software 2008; $27: 1-32$.

26. Racine J. Consistent significance testing for nonparametric regression. Journal of Business \& Economic Statistics 1997; 15: 369-78.

27. Racine JS, Hart J, Li Q. Testing the significance of categorical predictor variables in nonparametric regression models. Econometric Reviews 2006; 25: 523-44.

28. Hägg D, Eriksson M, Sundström A et al. The higher proportion of men with psoriasis treated with biologics may be explained by more severe disease in men. PloS one 2013; 8: e63619.

29. DeWitt EM, Lin L, Glick HA et al. Pattern and predictors of the initiation of biologic agents for the treatment of rheumatoid arthritis in the United States: an analysis using a large observational data bank. Clinical therapeutics 2009; 31: 1871-80.

30. Fraenkel L, Rabidou N, Dhar R. Are rheumatologists' treatment decisions influenced by patients' age? Rheumatology 2006; 45: 1555-7. 
Equality in the prescription of biologic medication in psoriasis care

31. Jones-Caballero M, Uaeze J, Penas PF et al. Use of biological agents in patients with moderate to severe psoriasis: a cohort-based perspective. Archives of dermatology 2007; 143: 846-50. 
Equality in the prescription of biologic medication in psoriasis care

Table 1: Description of variables by biologic initiation status

\begin{tabular}{|c|c|c|c|c|c|c|}
\hline Variable & Group & Proportion & Mean & SD & Min & Max \\
\hline \multirow[b]{2}{*}{ Age } & Treated & & 48.61 & 13.65 & 18.2 & 81.2 \\
\hline & $\begin{array}{l}\text { Not } \\
\text { treated }\end{array}$ & & 57.42 & 14.49 & 18.1 & 93.1 \\
\hline \multirow[b]{2}{*}{ Male } & Treated & $65.43 \%$ & & 0.48 & 0.0 & 1.0 \\
\hline & $\begin{array}{l}\text { Not } \\
\text { treated }\end{array}$ & $57.12 \%$ & & 0.50 & 0.0 & 1.0 \\
\hline \multirow[b]{2}{*}{ BMI } & Treated & & 28.12 & 5.61 & 16.0 & 52.2 \\
\hline & $\begin{array}{l}\text { Not } \\
\text { treated }\end{array}$ & & 27.50 & 5.33 & 14.2 & 80.0 \\
\hline \multirow{2}{*}{$\begin{array}{l}\text { Psoriatic } \\
\text { arthritis }\end{array}$} & Treated & $38.29 \%$ & & 0.49 & 0.0 & 1.0 \\
\hline & $\begin{array}{l}\text { Not } \\
\text { treated }\end{array}$ & $24.08 \%$ & & 0.48 & 0.0 & 1.0 \\
\hline \multirow[b]{2}{*}{ PASI } & Treated & & 11.46 & 8.12 & 0.0 & 67.8 \\
\hline & $\begin{array}{l}\text { Not } \\
\text { treated }\end{array}$ & & 6.89 & 5.79 & 0.0 & 41.8 \\
\hline \multirow[b]{2}{*}{$\mathrm{CCl}$} & Treated & & 0.08 & 0.44 & 0.0 & 4 \\
\hline & $\begin{array}{l}\text { Not } \\
\text { treated }\end{array}$ & & 0.05 & 0.33 & 0.0 & 3 \\
\hline \multirow{2}{*}{$\begin{array}{l}\text { Greater than } \\
\text { high school }\end{array}$} & Treated & $27.88 \%$ & & 0.45 & 0.0 & 1.0 \\
\hline & $\begin{array}{l}\text { Not } \\
\text { treated }\end{array}$ & $23.50 \%$ & & 0.42 & 0.0 & 1.0 \\
\hline \multirow{2}{*}{$\begin{array}{l}\text { Completed } \\
\text { high school }\end{array}$} & Treated & $52.79 \%$ & & 0.50 & 0.0 & 1.0 \\
\hline & $\begin{array}{l}\text { Not } \\
\text { treated }\end{array}$ & $49.92 \%$ & & 0.50 & 0.0 & 1.0 \\
\hline \multirow{2}{*}{$\begin{array}{l}\text { Less than high } \\
\text { school }\end{array}$} & Treated & $19.33 \%$ & & 0.40 & 0.0 & 1.0 \\
\hline & $\begin{array}{l}\text { Not } \\
\text { treated }\end{array}$ & $26.59 \%$ & & 0.44 & 0.0 & 1.0 \\
\hline
\end{tabular}

SD: Standard deviation, BMI: Body mass index, PASI: Psoriasis area and severity index, CCI: Charlson comorbidity index 
Equality in the prescription of biologic medication in psoriasis care

Table 2: Description of variables by registration year

\begin{tabular}{|c|c|c|c|c|c|c|}
\hline Variable & Group & Proportion & Mean & SD & Min & Max \\
\hline \multirow{3}{*}{ Treated } & 2007 & $38.84 \%$ & & 0.49 & 0.0 & 1.0 \\
\hline & 2008 & $18.89 \%$ & & 0.39 & 0.0 & 1.0 \\
\hline & 2009 & $10.80 \%$ & & 0.31 & 0.0 & 1.0 \\
\hline \multirow{3}{*}{ Age } & 2007 & & 54.83 & 15.13 & 18.6 & 90.3 \\
\hline & 2008 & & 56.67 & 13.88 & 18.1 & 87.5 \\
\hline & 2009 & & 55.34 & 15.33 & 18.62 & 93.1 \\
\hline \multirow{3}{*}{ Male } & 2007 & $60.71 \%$ & & 0.49 & 0.0 & 1.0 \\
\hline & 2008 & $58.69 \%$ & & 0.49 & 0.0 & 1.0 \\
\hline & 2009 & $57.87 \%$ & & 0.49 & 0.0 & 1.0 \\
\hline \multirow{3}{*}{ BMI } & 2007 & & 27.30 & 5.17 & 14.9 & 52.2 \\
\hline & 2008 & & 27.57 & 5.21 & 14.2 & 51.7 \\
\hline & 2009 & & 27.84 & 5.61 & 16.4 & 80.0 \\
\hline \multirow{3}{*}{$\begin{array}{l}\text { Psoriatic } \\
\text { arthritis }\end{array}$} & 2007 & $28.68 \%$ & & 0.45 & 0.0 & 1.0 \\
\hline & 2008 & $26.31 \%$ & & 0.44 & 0.0 & 1.0 \\
\hline & 2009 & $26.79 \%$ & & 0.44 & 0.0 & 1.0 \\
\hline \multirow{3}{*}{ PASI } & 2007 & & 8.29 & 6.46 & 0.0 & 32.4 \\
\hline & 2008 & & 7.36 & 6.37 & 0.0 & 41.8 \\
\hline & 2009 & & 7.88 & 6.77 & 0.0 & 67.8 \\
\hline \multirow{3}{*}{$\mathrm{CCl}$} & 2007 & & 0.10 & 0.50 & 0.0 & 4.0 \\
\hline & 2008 & & 0.05 & 0.30 & 0.0 & 3.0 \\
\hline & 2009 & & 0.06 & 0.34 & 0.0 & 4.0 \\
\hline \multirow{3}{*}{$\begin{array}{l}\text { Greater than } \\
\text { high school }\end{array}$} & 2007 & $31.25 \%$ & & 0.47 & 0.0 & 1.0 \\
\hline & 2008 & $20.91 \%$ & & 0.41 & 0.0 & 1.0 \\
\hline & 2009 & $25.00 \%$ & & 0.43 & 0.0 & 1.0 \\
\hline \multirow{3}{*}{$\begin{array}{l}\text { Completed } \\
\text { high school }\end{array}$} & 2007 & $46.43 \%$ & & 0.50 & 0.0 & 1.0 \\
\hline & 2008 & $51.60 \%$ & & 0.50 & 0.0 & 1.0 \\
\hline & 2009 & $50.77 \%$ & & 0.50 & 0.0 & 1.0 \\
\hline \multirow{3}{*}{$\begin{array}{l}\text { Less than high } \\
\text { school }\end{array}$} & 2007 & $22.32 \%$ & & 0.42 & 0.0 & 1.0 \\
\hline & 2008 & $27.49 \%$ & & 0.45 & 0.0 & 1.0 \\
\hline & 2009 & $24.23 \%$ & & 0.43 & 0.0 & 1.0 \\
\hline
\end{tabular}

SD: Standard deviation, BMI: Body mass index, PASI: Psoriasis area and severity index, CCI: Charlson comorbidity index 
Equality in the prescription of biologic medication in psoriasis care

Table 3: Cox proportional hazards model for time to initiation of biologic treatment -2007 stratification

\begin{tabular}{|l|c|c|c|c|}
\hline Variable & Hazard ratio & p-value & Lower CI & Upper Cl \\
\hline Age & 0.963 & $<0.005$ & 0.949 & 0.978 \\
\hline Male & 0.834 & 0.451 & 0.520 & 1.337 \\
\hline BMI & 0.999 & 0.983 & 0.960 & 1.041 \\
\hline Psoriatic arthritis & 1.363 & 0.187 & 0.860 & 2.16 \\
\hline PASI & 1.094 & $<0.005$ & 1.059 & 1.129 \\
\hline CCI & 1.245 & 0.267 & 0.846 & 1.831 \\
\hline Completed high school & 1.418 & 0.244 & 0.788 & 2.553 \\
\hline $\begin{array}{l}\text { Greater than high } \\
\text { school }\end{array}$ & 1.362 & 0.326 & 0.735 & 2.525 \\
\hline
\end{tabular}

Cl: Confidence interval, BMI: Body mass index, PASI: Psoriasis area and severity index, CCI: Charlson comorbidity index $N=224$, Biologic initiations $=87$ 
Equality in the prescription of biologic medication in psoriasis care

Table 4: Cox proportional hazards model for time to initiation of biologic treatment - 2008 stratification

\begin{tabular}{|l|c|c|c|c|}
\hline Variable & Hazard ratio & p-value & Lower Cl & Upper Cl \\
\hline Age & 0.968 & $<0.005$ & 0.955 & 0.982 \\
\hline Male & 1.038 & 0.851 & .702 & 1.536 \\
\hline BMI & 1.030 & 0.053 & 0.999 & 1.061 \\
\hline Psoriatic arthritis & 1.830 & 0.002 & 1.248 & 2.684 \\
\hline PASI & 1.051 & $<0.005$ & 1.028 & 1.076 \\
\hline CCI & 1.059 & 0.840 & .607 & 1.847 \\
\hline $\begin{array}{l}\text { Completed high } \\
\text { school* }\end{array}$ & 1.099 & 0.051 & .999 & 1.209 \\
\hline $\begin{array}{l}\text { Greater than high } \\
\text { school }\end{array}$ & 1.714 & 0.029 & 1.058 & 2.777 \\
\hline
\end{tabular}

Cl: Confidence interval, BMI: Body mass index, PASI: Psoriasis area and severity index, CCI: Charlson comorbidity index $N=593$, Biologic initiations $=112$

${ }^{*}$ Time varying-coefficient: Interacted with In(time) 
Equality in the prescription of biologic medication in psoriasis care

Table 5: Cox proportional hazards model for time to initiation of biologic treatment - 2009 stratification

\begin{tabular}{|l|c|c|c|c|}
\hline Variable & Hazard ratio & p-value & Lower CI & Upper Cl \\
\hline Age & 0.969 & $<0.005$ & 0.953 & 0.985 \\
\hline Male & 1.620 & 0.073 & 0.957 & 2.745 \\
\hline BMI & 1.021 & 0.237 & 0.987 & 1.056 \\
\hline Psoriatic arthritis & 2.063 & 0.004 & 1.263 & 3.370 \\
\hline PASI & 1.078 & 0.000 & 1.056 & 1.102 \\
\hline CCI & 1.241 & 0.451 & 0.708 & 2.175 \\
\hline Completed high school & 1.017 & 0.959 & 0.532 & 1.943 \\
\hline $\begin{array}{l}\text { Greater than high } \\
\text { school }\end{array}$ & 0.978 & 0.955 & 0.461 & 2.072 \\
\hline
\end{tabular}

Cl: Confidence interval, BMI: Body mass index, PASI: Psoriasis area and severity index, CCI: Charlson comorbidity index $N=648$, Biologic initiations $=70$ 